\title{
Acute Pulmonary Embolism in a Teenage Female - A Case Report
}

\author{
Iulia Armean¹, Lorena Elena Meliț1,2*, Iunius Simu³ ${ }^{3}$ Carmen Duicu1,2 \\ 1 Emergency Clinical County Hospital Tîrgu Mureș, Pediatrics Clinic No. 1, Tîrgu Mureş, Romania \\ 2 University of Medicine and Pharmacy Tîrgu Mureș, $1^{\text {st }}$ Department of Pediatrics, Tîrgu Mureş, Romania \\ 3 Emergency Clinical County Hospital Tîrgu Mureș, Radiology Department, Tîrgu Mureş, Romania
}

\begin{abstract}
Thrombophilia represents a tendency towards excessive blood clotting and the subsequent development of venous thromboembolism (VTE). VTE is a rare condition in children that comprises both deep venous thrombosis (DVT) and pulmonary embolism (PE). This paper reports the case of a 16-year-old girl, admitted to the Pediatrics Clinic No. 1, Tîrgu Mureș, Romania, for dyspnea, chest pain and loss of consciousness. Her personal history showed that she had had two orthopedic surgical interventions in infancy, two pregnancies, one spontaneous miscarriage and a recent caesarian section at 20 weeks of gestation for premature detachment of a normally positioned placenta associated with a deceased fetus. Laboratory tests showed increased levels of D-dimers. Angio-Computed Tomography (Angio$\mathrm{CT}$ ) showed multiple filling defects in both pulmonary arteries, establishing the diagnosis of PE. The laboratory tests were undertaken to assist in the diagnoses of a possible thrombophilia underlined a low level of antithrombin III. Antiphospholipid syndrome was ruled out and genetic tests revealed no specific mutation. Anticoagulant therapy was initiated with unfractionated heparin and afterwards subcutaneously low molecular heparin was prescribed for three months. Later it has been changed to oral therapy with acenocoumarol. The patient was discharged in good general status with the recommendation of life-long anticoagulation therapy. Thrombophilia is a significant risk factor for PE, and it must be ruled out in all cases of repeated miscarriage.
\end{abstract}

Keywords: thrombophilia, pulmonary embolism, children

Received: 25 June 2018 / Accepted: 26 July 2018

\section{-INTRODUCTION}

Thrombophilia is defined as a tendency towards excessive blood clotting [1]. The inherited types of thrombophilia include prothrombin (PT) gene (G20210A) mutation, factor V Leiden (FVL) mutation, protein C (PC) deficiency, protein S (PS) deficiency, and antithrombin III (ARIII) deficiency [2]. This hypercoagulable state, either acquired or inherited, increases the risk of developing venous thromboembolism (VTE) [2]. Other frequent causes that may lead to VTE in pediatric patients are vascular malformation or sepsis [3-5].

VTE, which comprises deep vein thrombosis (DVT) and its life-threatening complication, acute pulmonary embolism (PE), represents a significant worldwide health problem which can result in death. The annu- al incidence of VTE ranges between 75 and 269 cases per 100,000 individuals, as shown by global studies in Western Europe, North America, Australia, and southern Latin America [6,7]. In the United States, PE is a relatively common disease, with an incidence ranging from 60 to 112 per 100,000 inhabitants [8].

Recent studies using CT pulmonary angiography have proved a high incidence (14-15.5\%) of PE in children [9]. PE in the pediatric population is rare, but evidence suggests it often goes undetected and seldom considered, as part of a differential diagnosis, by physicians. Predisposing risk factors for PE in children include obesity, immobility, central venous catheter, malignancy, congenital heart disease, systemic lupus erythematosus, nephrotic syndrome, surgery, trauma, and prolonged total parenteral nutrition [10]. The early 
diagnosis and treatment of PE are essential, while failure to diagnose the condition can have severe consequences. Mortality rates of $\mathrm{PE}$ in childhood are reported to be up to $10 \%$, in a Canadian registry $[10,11]$.

Pregnancy is a physiological state that predisposes to thrombosis due to hormonal changes that involve blood flow (venous stasis), the vascular wall (hypotonia, endothelial lesion), coagulation factors (increased levels of factor VII, factor VIII, factor X, von Willebrand factor) and decreased activity levels of natural anticoagulants (protein C, protein S) [12]. Also, in some cases, this physiological hypercoagulable state can be augmented by hereditary or acquired 'defects' in anticoagulant mechanisms. [12,13] VTE during pregnancy has an incidence of $1 / 1000$ births. The risk of developing VTE is 4.5 times higher in pregnant compared to non-pregnant women. $75-80 \%$ of VTEs related to pregnancy are deep DVTs and 20-25\% are Eps. [12-14] Thrombophilia is a common cause of recurrent pregnancy loss representing up to $40-50 \%$ of cases. [1]

\section{CASE REPORT}

\section{Medical history}

The case describes a 16-year-old girl, admitted to the Pediatrics Clinic, for dyspnea, sudden onset chest pains and one episode of loss of consciousness. Her medical history revealed two orthopedic surgical interventions in infancy for inferior limb inequality, two pregnancies, one spontaneous miscarriage one year before this admission. One week before this current admission, she had undergone a caesarian section at 20 weeks of gestation for the premature detachment of a normally

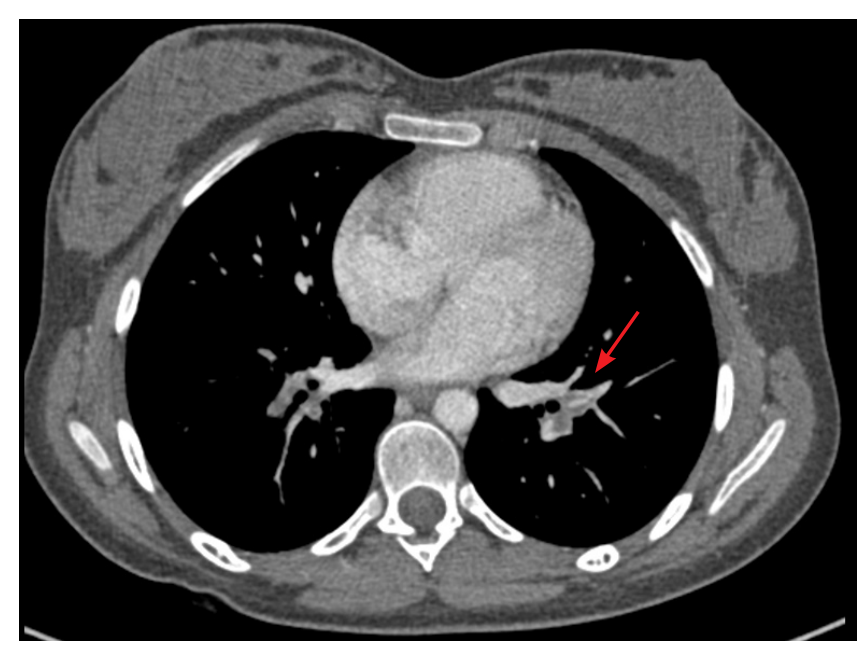

Fig. 1. CT-scan: Thrombus in the left pulmonary artery situated placenta associated with a deceased fetus. The patient is a non-smoker, does not consume alcohol or other drugs, and has not used oral contraceptives. Her family history did not reveal any relevant pathological elements.

\section{Clinical findings}

On admission, the clinical examination showed the following pathological elements: altered general health status, ringed face, pallor, sweaty skin, tachycardia, a post-cesarean scar in the lower abdominal quadrant, inferior limb inequality with the right inferior limb shorter by 8-10 centimeters. Examination of the respiratory tract revealed symmetrically disseminated sibilant rales and an $\mathrm{O}_{2}$ saturation in hemoglobin $\left(\mathrm{SaO}_{2}\right)$ of $94 \%$. Weight $59 \mathrm{~kg}$, height $164 \mathrm{~cm}$, and a body mass index of 22.01 .

\section{Diagnosis}

The pathological elements of the laboratory tests consisted of low activated partial thromboplastin time (APTT) 21.9 seconds. The international normalized ratio (INR) was 1.01. An angio-CT was performed which showed multiple filling defects in both pulmonary arteries, mixed thrombi, both floating and parietal, positioned in the area of pulmonary artery dichotomy with extension in the bilateral lower lobar artery and bilaterally in the lower lobe segmental arteries. The right arterial lumen was completely obstructed and the left arterial lumen partially obstructed (Figure 1,2). Laboratory tests were performed in order to diagnose a possible thrombophilia. The results indicated antithrombin III $30 \%$, repeated $16.7 \%$, C protein $107.7 \%$, S protein $78.6 \%$, and homocysteine $10.14 \mu \mathrm{mol} / \mathrm{l}$. Genetic testing

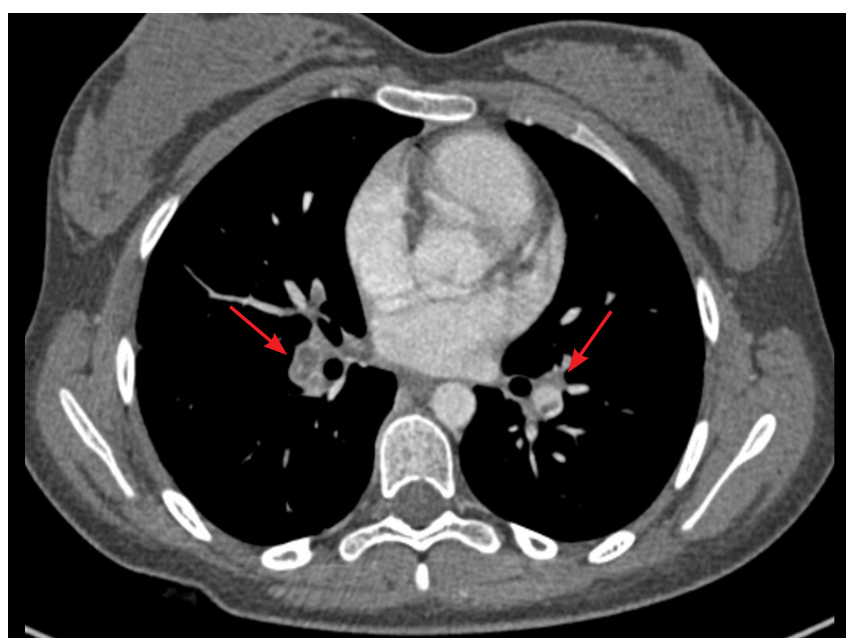

Fig. 2. CT-scan: Partial obliteration of both pulmonary arteries 
revealed no mutation of factor $\mathrm{V}$ Leiden (A506G) or factor II (prothrombin gene G20210A). Antiphospholipid syndrome was ruled based on lupus anticoagulant, anti-cardiolipin antibodies and beta2-glycoprotein I Ig $\mathrm{M}$, IgG, that were within normal ranges. Based on these data, a diagnosis of PE and thrombophilia (antithrombin III deficiency) was made.

\section{Therapy}

Anticoagulant therapy with 5000 UI unfractionated heparin as a bolus, followed by 20000 UI in perfusion, with close monitoring of the coagulation parameters was prescribed. The treatment was continued for five days with 50000 UI unfractionated heparin. Antibiotic therapy with amikacin $(2 \times 500 \mathrm{mg} /$ day, intra venous, 9 days) and ceftazidime ( $3 \times 1 \mathrm{~g} /$ day, intra venous, 13 days) was prescribed. Subsequently, the patient received low molecular weight heparin $0,6 \mathrm{ml}$ (100UI/ $\mathrm{kg}$ ) subcutaneously for three months. This approach was taken because the patient refused INR monitoring. Unfortunately, she developed an exanthema with pruritus after the administration of Fraxiparine, which was considered as a potential allergic reaction to the therapy. Therefore, the subcutaneous anticoagulant treatment was replaced with acenocoumarol $(3 \mathrm{mg} /$ day, oral, life-long) and close monitoring of INR.

\section{Discussion}

$\mathrm{PE}$ is a difficult to diagnose, life-threatening condition presenting with non-specific signs and symptoms. [8] Mortality rates of $\mathrm{PE}$ during childhood are reported to reach approximately $10 \%$. A recent study found the incidence of clinically relevant $\mathrm{PE}$ to be of 25 cases per 100000 admissions. [10]. Despite known predisposing risk factors and associated signs, and symptoms, the diagnosis of PE is still often delayed because the presenting disorders may overlap with other conditions.[10]

VTE is a major cause of maternal morbidity and mortality during pregnancy or early after delivery, and remains a diagnostic and therapeutic challenge in both circumstances. [15] The incidence of pregnancy-associated VTE has been reported to be between 1 in 1,000 and 1 in 2,000 deliveries. Also, it was found that $20 \%$ of these episodes are due to arterial events, while $80 \%$ are venous. VTE episodes are 4-5-fold higher during pregnancy than in a non-pregnant status. Moreover, it was proved that VTE risk is higher in the postpartum than the antepartum period. A 20- to 80-fold higher VTE risk has been reported within the first six weeks following delivery, being 100-fold higher in the first week after delivery [15].

The most common clinical indicators of PE in pregnancy include shortness of breath, pleuritic chest pain, hypoxemia, and tachycardia. Among the less frequently reported symptoms are tachypnea, hemoptysis, syncope, cough, unexplained hypotension, and other types of chest pain. [16] The patient in this reported case presented with several of the signs mentioned above i.e. sudden onset chest pain and one episode of syncope.

Pregnancy impairs the hemostatic system leading to a state of hypercoagulability which increases in severity during pregnancy and reaches the maximum point around term. These peri-partum changes in the hemostatic system may lead to maternal and fetal complications during pregnancy. The mother is at risk from the time of conception until the postnatal period, with recent data suggesting that the risk extends to at least 12 weeks postpartum. The fetal risks include pre-eclampsia, placental abruption, fetal growth restriction, late and recurrent early miscarriage, intrauterine death and stillbirth. [17]

Beyond the specific pregnancy-related increased hemostatic activation, congenital or acquired thrombophilia are frequently encountered, accounting for approximately $15 \%$ of all thrombophilic conditions in western countries and up to 50\% of VTE cases during pregnancy or the postpartum period. $[15,10]$ There is a paucity of strong evidence associated with adverse pregnancy outcomes and thrombophilia in pregnancy. These problems include both early and late placental vascular-mediated problems. [17-19]

Clinical studies suggest that hyper coagulation is the primary underlying pathophysiological mechanism which leads to uteroplacental insufficiency and, subsequent miscarriage. It is considered that thrombophilia impairs the placental function by causing arterial and venous thrombosis at the maternal-fetal interface. $[1,19]$ Similarly, in the current case, there was one miscarriage and a recent caesarian section at the gestational age of 20 weeks.

\section{CONCLUSIONS}

Thrombophilia is a disorder of hemostasis that predisposes a person to a thrombotic event. The association between thrombophilia and recurrent pregnancy loss has become an undisputed fact. VTE has an increased 
104 The Journal of Critical Care Medicine 2018;4(3)

incidence among pregnant women, but the highest risk is observed postpartum. The case presented above emphasized a female teenager associated with an increased number of risk factors which contributed to PE development. The importance of ruling out thrombophilia in all cases of repeated miscarriage is emphasized as this hypercoagulable state representing a critical risk factor for PE.

\section{CONFLICT OF INTEREST}

None to declare

\section{REFERENCES}

1. Abu-Heija A. Thrombophilia and Recurrent Pregnancy Loss. Is heparin still the drug of choice?. Sultan Qaboos University Med J. 2014;14:26-36.

2. Adetola FLJ, Maggio L, Romero ST. Prenatal Screening for Thrombophilias Indications and Controversies, an Update. Clin Lab Med. 2016;36:421-34.

3. Armean I, Duicu C, Aldea C, Melit LE. Serratia marcescens Sepsis in a Child with Deep Venous Thrombosis - A Case Report. J Crit Care Med (Targu Mures). 2018;4:29-33.

4. Duicu C, Bucur G, Simu I, Marginean O. Deep Venous Thrombosis Associated With Inferior Vena Cava Abnormalities And Hypoplastic Kidney In Siblings. Acta Medica Marisiensis. 2016;62:266-268

5. Meliț LE, Mărginean CO, Georgescu A, Duicu C. Complications of sepsis in infant. A case report. J Crit Care Med (Targu Mures). 2016;2:96-9.

6. Konstantinides SV, Barco S, Lankeit M, Meyer G. Management of Pulmonary Embolism An Update. J Am Coll Cardiol. 2016;67:976-89.

7. Raskob GE, Angchaisuksiri P, Blanco AN, et al. Thrombosis: a major contributor to global disease burden. Arterioscler Thromb Vasc Biol. 2014;34:2363-71.

8. Meyer G. Effective diagnosis and treatment of pulmonary
Available online at: www.jccm.ro embolism: Improving patient outcomes. Arch Cardiovas Dis. 2014;107:406-14.

9. Tang CX, Schoepf UJ, Chowdhury SM, et al. Multidetector computed tomography pulmonary angiography in childhood acute pulmonary embolism. Pediatr Radiol. 2015;45:1431-39.

10. Agha SB, Sturm JJ, Simon HK, Hirsh AD. Pulmonary Embolism in the Pediatric Emergency Department. Pediatrics. 2013;132:663-7.

11. Ommen VCH, Heijboer $H$, Buller $H R$, et al. Venous thromboembolism in childhood: a prospective two year registry in the Netherlands. J Pediatr. 2001;139:676-81.

12. Coriu LU, Talmaci R, Uscatescu V, et al. Hereditary Thrombophilia and thrombotic events in pregnancy: singlecenter experience. J Med Life. 2014;7:567-71.

13. Martinelli I, Battaglioli T, De Stefano V, el al. The risk of first venous thromboembolism during pregnancy and puerperium in double heterozygotes for factor $V$ Leiden and prothrombin G20210A. Journal Thromb Haemost. 2007;6:494-8.

14. Bates SM, Greer IA, Middeldorp S. VTE, thrombophilia, antithrombotic therapy, and pregnancy: Antithrombotic therapy and prevention of thrombosis. 9th ed. American College of Chest Physicians evidence-based clinical practice guidelines. Chest. 2012;141:691-736.

15. Conti E, Zezza L, Ralli E, et al. Pulmonary embolism in pregnancy. Journal Thromb Thrombolysis. 2014;37:251-70.

16. Cahill AG, Stout MJ, Macones GA, et al. Diagnosing pulmonary embolism in pregnancy using computed-tomographic angiography or ventilation-perfusion. Obstet Gynecol. 2009;114:124-9.

17. Simcox EL, Ormesher L, Tower C, Greer IA. Thrombophilia and Pregnancy Complications. Int J Mol Sci. 2015;16:28418-28.

18. Martínez-Zamora MA, Cervera R, Balasch J. Recurrent Miscarriage, Antiphospholipid Antibodies and the Risk of Thromboembolic Disease. Clinic Rev Allerg Immunol. 2012;43:265-74.

19. Aksoy M, Tek I, Karabulut H, Berker B, Soylemez F. The role of thrombofilia related to Factor $\mathrm{V}$ Leiden and Factor II G20210A mutations in recurrent abortions. J Pak Med Assoc. 2005;55:104-8. 